\title{
Radiation Synthesis and Characterization of Cholesterol Molecularly Imprinted Polymer of Crosslinked Hydroxyethyl Methacrylate
}

\author{
EL-Sayed A. Hegazy ${ }^{1}$, Gilane M. Sabry ${ }^{2}$, Magda K. Ezz ${ }^{3}$, H. Kamal ${ }^{4}$, S. Lotfy ${ }^{5}$, Somaya Zakaria Mansour \\ Dina Hassan Eissa ${ }^{7}$
}

${ }^{1}$ Polymer Chemistry Department, National Center for Radiation Research and Technology (NCRRT), Atomic Energy Authority (AEA), PO Box 29, Nasr City, Cairo, Egypt.

${ }^{2}$ Biochemistry Department, Faculty of Science, Ain Shams University, Cairo, Egypt.

${ }^{3}$ Biochemistry Department, Faculty of Science, Ain Shams University, Cairo, Egypt.

${ }^{4}$ Polymer Chemistry Department, National Center for Radiation Research and Technology (NCRRT), Atomic Energy Authority (AEA), PO Box 29, Nasr City, Cairo, Egypt.

${ }^{5}$ Polymer Chemistry Department, National Center for Radiation Research and Technology (NCRRT), Atomic Energy Authority (AEA), PO Box 29, Nasr City, Cairo, Egypt.

${ }^{6}$ Radiation Biology Department, National Centre for Radiation Research and Technology, Atomic Authority, Cairo, Egypt.

${ }^{7}$ Radiation Biology Department, National Centre for Radiation Research and Technology, Atomic Authority, Cairo, Egypt.

\begin{abstract}
Cholesterol-imprinted poly hydroxyethyl methacrylate (MIP) andnon-imprinted polyhydroxyethyl methacrylate (NIP) have been prepared via polymerization by gamma radiation. The functional structure of the imprinted and non-imprinted polymers were characterized by Fourier Transform Infrared spectroscopy (FT-IR); the spectrum showed that the HEMA interacts with cholesterol through H-bonds. The surface morphology of NIP and MIP were investigated by scanning electron microscope (SEM) and the MIP image appeared as a rough surface, while NIP had no pores. The specificity of MIP to interact with the print molecular was studied cholesterol and the results showed a higher cholesterol adsorption by MIP than that by NIP. This work approachesto design robust MIP to mimic natural recognition entities as adsorbed materials of cholesterol.
\end{abstract}

Keywords: Molecular imprinted polymers, NIP, HEMA, Cholesterol and Gamma Radiation

\section{Introduction}

Cholesterol takes a significant part in many biological mechanisms and it is as important componentsinthe manufacture of bile acids, steroid hormones, and several fatsoluble vitamins[1]. High levels of blood cholesterol increase the risk of heart disease[2]. Cholesterol is a steroid that plays important roles in developing cardiovascular diseases. Correctly quantified cholesterol concentration on biological and food samples is in great need, especially for patients sufferingfrom heart diseases[3-4].It is involved in the atherosclerosis development and in heart degenerative processes. Cholesterol is also a constituent of animal foods such aseggs, meat and dairy products [5].Determination of cholesterol content in food is of primaryimportance to select a diet with low intake of cholesterol.In the analytical practice, well known GC and HPLCmethods are used for the cholesterol measurement, but avery intensive pre-treatment of the samples is needed [6].In the last few years a lot of research has gone intoestablishing rapid routine methods for the fast determinationof cholesterol.The most common and cheapest purification techniquesare thin-layer chromatography (TLC) and Solid-PhaseExtraction (SPE) [7].Molecular imprinting Technology (MIT) is today a viable synthetic approach to design robust molecular recognition materials able to mimic natural recognition entities, such as antibodies and biological receptors[8].Cholesterol is the attractive target molecule in the clinical field, therefore, it was used as the target imprinting molecule (template) in previous arts .Cholesterol template possesses a conformational rigidity can fit well in the cavity of the polymer matrix with minimal change in conformation and will increase the affinity and selectivity in the recognition [9-10].

Molecular imprinting is a powerful method, which provides synthetic polymers with specific binding properties. The synthesis of MIP involves the assembly crosslinking of monomers around a template molecule followed by polymerization in the presence of a cross linker. "Removal of the template molecule by extraction leaves sites specific to the template molecule in both a shape and chemical functionality, thus enabling subsequent recognition of the template"[11].MIPs are well known for their specificity in the interaction with the print molecules. The efficiency of imprinting is evaluated by studying the interaction of MIP with another molecule of close resemblance between geometry and structure with the print molecule. The uptake of this molecule should be less, compared to the extent of absorption the print molecule [12]. 


\section{International Journal of Science and Research (IJSR) \\ ISSN (Online): 2319-7064 \\ Index Copernicus Value (2013): 6.14 | Impact Factor (2014): 5.611}

Molecularly imprinted polymers are crosslinked polymeric materials that exhibit high binding capacity and selectivity against a target molecule (template) purposely present during the synthesis process. In solutions, the template interacts with functional monomers or precursors and the structure of these aggregates or complexes is preserved by copolymerization in the presence of an excess amount of a cross linker. After polymerization, the template is removed from the polymer matrix, thus leaving internal images with specific binding sites in the material. In the mixture, prior to polymerization, the dissolved template may interact by covalent, non-covalent or by metal coordination interactions with the functional monomer. "Consequently, molecular imprinting may be classified into covalent imprinting (preorganized), non-covalent imprinting (self-assembly) and semi-covalent imprinting, according to the type of interactions involved between the functional monomers and the template in the pre-polymerization mixture and during rebinding"[13]. This study aims to prepare molecular imprinted polymers with high crosslinking to be used in cholesterol adsorption.

\section{Material and Experiment}

\subsection{Materials}

Hydroxyethyl methacrylate (HEMA) was purchased from Mark-Schuchardt (Germany), Ethylene glycol dimethacrylate (EGDMA) and cholesterol powder were purchased from Alfa Aeasar (Germany),methanol purity 95\%(AR) andchloroformpurity 99\% (AR), were purchased from Sigma (USA). All chemicals were used as received.

\subsection{Preparation of Cholesterol imprinted polymers (MIP) and non-imprinted polymers (NIP)}

Hydroxyethyl methacrylate (HEMA) was used as functional monomers to prepare the MIP by the non-covalent imprinting method. Briefly, template cholesterol, HEMA and EGDMA were dissolved in a mixture of methanol and chloroform in a thick-walled glass tube. The tube was purged with nitrogen, sealed and subjected to gamma irradiation from a $\mathrm{Co}^{60}$ source $\left(\mathrm{Co}^{60}\right.$ Indian GC type $\gamma$ irradiator).

HEMA was polymerized under the same conditions without cholesterol to serve as a control (NIP) [14]. After copolymerization, the cylindrical shape gel, were cut into discs of 2-3 mm thickness and dried to constant weight. All samples were washed in excess water to remove the unreacted component, then air-dried up to a constant weight at room temperature [15].

\subsection{Characterization of NIP and MIP}

\subsubsection{Gel content of MIP and NIP}

The gel content of the MIP and NIP were determined by extracted in distilled water for 24 hours of $70{ }^{\circ} \mathrm{C}$ with shaking. The insoluble parts corresponding to the crosslinking sample were dried till constant weight. The gel content percent was calculated according to the equation (1). Gel content $\%=(\mathrm{Ws} / \mathrm{Wo}) \times 100$
Where Ws is the weight of the insoluble dry parts after extraction andWo is the initial weight of the dry gel.

\subsubsection{Fourier Transform Infrared (FT-IR) spectroscopy} FTIR spectra of cholesterol, NIP, MIP (After elimination of Cholesterol template) and MIPs were measured by using FTIR spectrophotometer (JASCO FT- IR-6300, Japan). The dry grind sample of $0.5 \mathrm{~g}$ was thoroughly mixed with $\mathrm{KBr}$ (0.1 g, IR Grade, Merck, Germany), and pressed in a pellet form and the FTIR spectrum was then recorded.

\subsubsection{Scanning Electron Microscope (SEM)}

The surface morphology of the samples was examined usingscanning electron microscopy (SEM). The MIPs, NIPs samples were allowed to swell till equilibrium. Samples were initiallydried in the air at room temperature for 7 days before being analyzed. Afragment of the dried samples was mounted on an SEM samplemount, freeze dried, and then the surfaces were pre-coated with a thin gold layer to reduce charging. The samples thenmounted on a SEM (model JSM5400 JEOL, Japan). They were then scanned at the desired magnification to study the samples [16].

\subsection{Removal of Template Molecules}

In order to remove the unreacted monomers and other ingredients, the (MIP and NIP) were extensively washed with (methanol/water)solution (70:30)for 24 hours at room temperature. After washing, the template was removed from the polymers using (chloroform: ethanol: acetic acid) (3:1:1)Where, MIP was added into $10 \mathrm{ml}$ of (chloroform: ethanol: acetic acid) (3:1:1)for 48 hours. This procedure was repeated until no cholesterol leakage was observed from MIP to the wash solution. [17-18]. The amount and concentration of cholesterol, extracted from MIPin aqueous solutions were estimated and measured by using Liebermann-Burchard reagent [19].

\subsection{Cholesterol adsorption studies}

Adsorption of cholesterol molecules on the MIP and NIP were carried out using a batch wise adsorption method [20].To observe the adsorption of cholesterol onto the free cholesterol eluted MIP,the MIP and NIPwere placed in aqueous solutions to different concentrations of cholesterol. The amount of cholesterol bound to imprint was calculated according to equation (1) and estimated by the Lieberman Burchard method, then measured by a UV-Visible Spectrophotometer $\lambda$ max at $610 \mathrm{~nm}$. The amount of bounds cholesterol was calculated using the following equation:

$$
\mathrm{Q}=\left[\left(\mathrm{C}_{\mathrm{i}}-\mathrm{C}_{\mathrm{r}}\right) \times \mathrm{V}\right] / \mathrm{W}
$$

Where Qis the amount of bound cholesterol onto the gel ( $\mathrm{mg}$ $\mathrm{g}^{-1}$ ), $\mathrm{Ci}$ and $\mathrm{C}_{\mathrm{r}}$ are the concentration of the initial and final cholesterol in the reaction medium. $\mathrm{V}$ is the volume of the aqueous solution of the reaction medium. $\mathrm{W}$ is the weight of the MIP. All data used in this formula are averages of at least duplicated experiments [21].

\subsection{Selectivity studies}

It is important to study the selectivity of MIPs and NIPs toward cholesterol and testosterone. For this purpose, the MIPs and NIPs are immersed in cholesterol and testosterone 


\section{International Journal of Science and Research (IJSR) \\ ISSN (Online): 2319-7064}

Index Copernicus Value (2013): 6.14 | Impact Factor (2014): 5.611

solutions. A mixture of equal concentration (cholesterol and testosterone) solutions were added to $0.1 \mathrm{~g}$ MIPsThe samples were shaken for $24 \mathrm{~h}$. The MIPs and NIPs removed from the solution then the remaining concentration of cholesterol and testosterone in the supernatant was analyzed by Random access immunoassay system (ADVIAcentaur, Germany).

\section{Result and Discussion}

\subsection{Characterization Studies}

\subsubsection{Effect of monomer concentration and irradiation dose on gel content}

In the presence of monomer with high polymerization tendencies such as hydroxyethyl methacrylate, it is randomly incorporated into the main chain [22]. When an aqueous solution of monomer is irradiated with gamma rays, the radiolysis products of water, especiallygel free radicalsare attacking the monomer and the polymeric network is formed [23].It is clear that, the gel content of NIPincreases in increasing the irradiation dose and monomer concentration. At low irradiation doses and low monomer concentration, the network consists of polymer chains jointed through multifunctional junctions, thus forming giant molecules with branches. By increasing both monomer concentration and irradiation dose,the polymer chains are crosslinked and polymeric network are obtained [24].The obtained results may be due to the higher crosslinking at the high irradiation dose and monomer concentration.

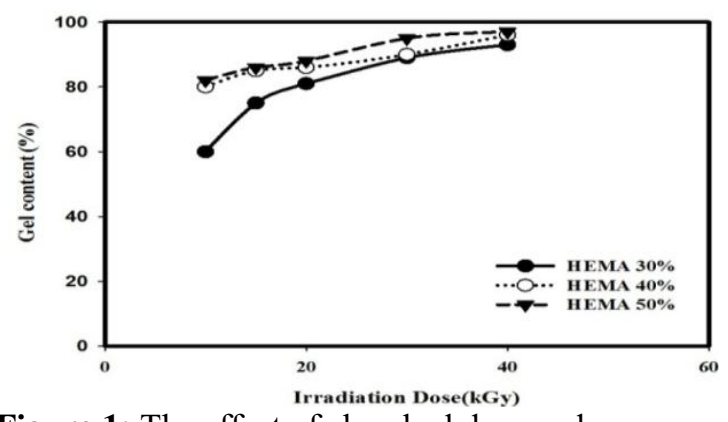

Figure 1: The effect of absorbed dose and monomer concentration on the gel content percent of NIP, at a cross linker (EGDMA) concentration of $1 \mathrm{wt} \%$.

The comparison between the gel fraction percent of NIP and MIP show that, the gel content percent of NIP found to be slightly higher than MIP.The gel content of cholesterol MIP is lower than that of its NIP, whereas the functional monomer HEMA used for producing a useful MIP is supposed to be strongly interacting with the cholesterol template to achieve a high yield of imprinted binding sites and allow the maximum number of complementary interactions to be developed in the polymer matrix, so this is due to higher affinity of HEMA to crosslinking through the formation of intra and/or intermolecular hydrogen bonding via $-\mathrm{OH}$ groups. These results may be attributed to the high crosslinking of the free groups of HEMA than that of HEMA imprinted cholesterol, which leads to form a high crosslinking polymeric network as shown in figure 2 .

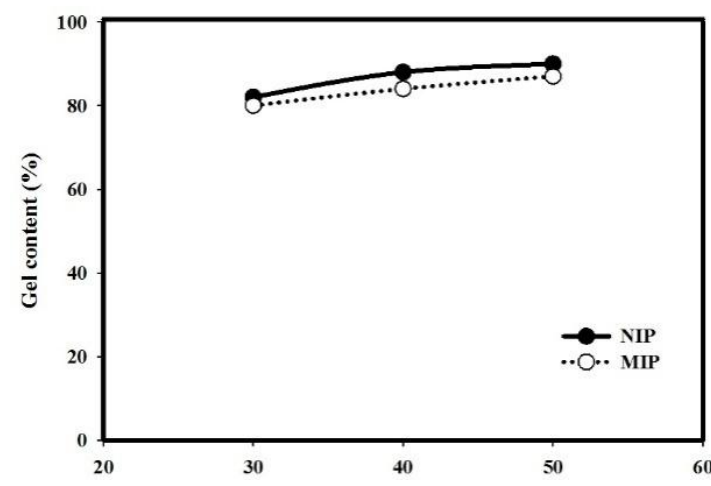

Figure 2: The effect of Monomer (HEMA) concentration of NIP and MIP on gel content percent, Irradiation dose of 20 $\mathrm{kGy}$, Cholesterol concentration of $200 \mathrm{~m} / \mathrm{dl}$, at a cross linker (EGDMA) concentration of $1 \mathrm{wt} \%$.

\subsubsection{Fourier transfer infrared (FTIR analysis)}

FTIR spectra of prepared materials, to highlight the trail of imprinting, NIP, cholesterol template and MIP spectra. There are three main absorption bands (3200-3600, 29003100 and $\sim 1750 \mathrm{~cm}^{-1}$ ). These main absorption bands, as already displayed, can be related to hydroxyl, $\mathrm{C}-\mathrm{H}_{3}$ and carbonyl groups. Some overlapped peaks appearing in these spectraNIP, cholesterol templates and MIP have the characteristic peaks corresponding to $\mathrm{OH}$ bands at $3417 \mathrm{~cm}$ ${ }^{1}, \mathrm{C}-\mathrm{O}-\mathrm{C}$ band at $1164 \mathrm{~cm}^{-1}$ and carbonyl band at $1720 \mathrm{~cm}^{-1}$. FTIR spectrum of pure cholesterol includes $\mathrm{H}$ bonded alcohol at $3417 \mathrm{~cm}^{-1}$ and strong hydrocarbon peaks around $2950 \mathrm{~cm}^{-1}$ and $1466 \mathrm{~cm}^{-1}[25]$ meanwhile the characteristic band corresponding to $\mathrm{C}=\mathrm{O}$ at $1720 \mathrm{~cm}^{-1}$ disappeared in cholesterol FTIR spectra. HEMA was interacted with cholesterol through H-bonds. FTIR spectrum of MIP exhibited shifts in wave numbers of related peaks.As noted, there is a similar existing on the spectra of NIP, MIP. After washing, the spectrum of the MIP becomes comparable to that of the blank polymer. This indicates that almost all templates are removed from the precursor, which therefore presents an advantage for the study of binding behaviors.

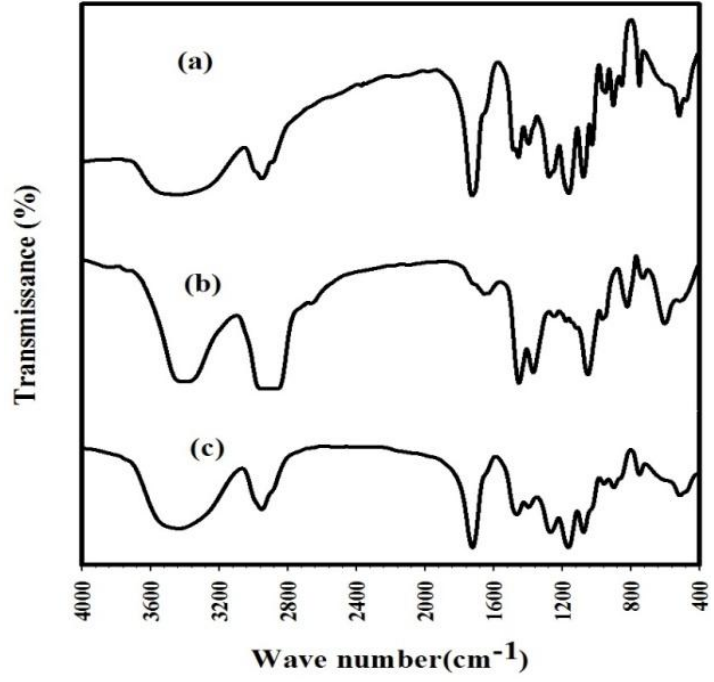

Figure 3: FT-IR spectra for: (a) NIP, (b) Cholesterol template, (c) MIP

\subsubsection{Scanning Electron Microscope}

The surface morphology and internal structure of thecholesterol template, NIP, MIP, MIP after adsorption of 


\section{International Journal of Science and Research (IJSR) \\ ISSN (Online): 2319-7064}

Index Copernicus Value (2013): 6.14 | Impact Factor (2014): 5.611

cholesterol and MIP after elimination of cholesterol template. Theimagesshowed that MIPhas a rough surface due to the pores, which formed during the polymerizationprocess in the presence of cholesterol template; otherwiseNIPwas inert with no pores as the polymerization ingredients doesn't contain cholesterol. When the cholesterol was eliminated from MIP, the pores tend to be connected to each other, form some open channels, which could remarkably facilitate the adsorption and release of cholesterol in and out of the MIP. The polymerization ingredients were well organized around cholesterol molecules through secondary interactions to make cholesterol cages, these results confirmed that cholesterol templates deposited and adsorbed by eluted MIP [17]. The morphology of the MIP after elimination of cholesterol template indicates that almost all templates are removed from the precursor, which therefore presents an advantage for the study of binding behaviors. As noted, the morphology of MIP after adsorption of cholesterol becomes similar comparable to that of MIP.

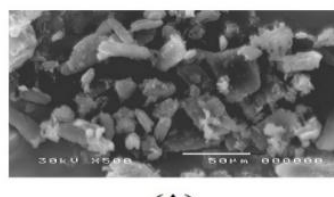

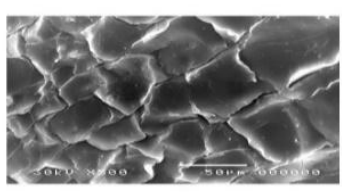

(B)

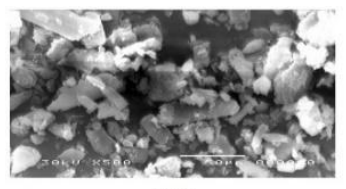

(D)

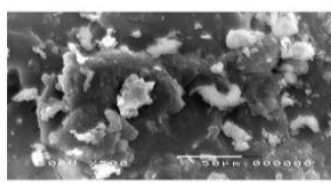

(C)

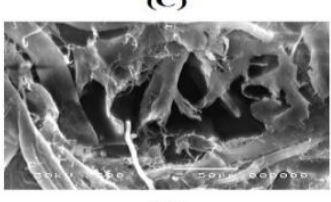

(E)
Figure 4:SEM micrographs for: (A) Cholesterol template, (B) NIP, (C) MIP, (D) MIP (after adsorption of cholesterol),

(E) MIP (after elimination of cholesterol template).

\subsection{Cholesterol leakage and elution studies}

The amount of cholesterol inwashingsolution was estimated. It isfound that the cholesterol release percent was increased by time also the cholesterol releases percent inchloroform/ethanol/ acetic acidas an eluting system, reached $90.5 \%$ followed by chloroform. Meanwhile, on using both methanol/acetic acid and acetonitrile/waterthe cholesterol release reached its maximum value after 9 hours only as shown in figure 5. Cholesterol templates removal from the prepared MIP created the cavities capable of cholesterol specific sorption. This may be due to the effect of the residues of hydroxyl groups of the HEMAwhich give a hydrophilic property to the crosslinked polymer network and this property would ease the separation of cholesterol from molecularly imprinted polymers [17], so the removal of cholesterol may be obtained by hydrolysis.

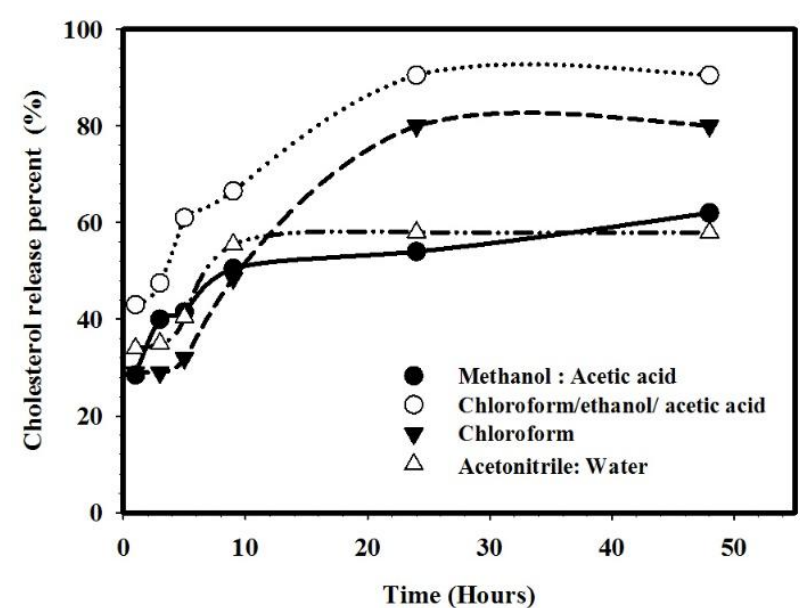

Figure 5: Effect of time of release and removal of cholesterol from MIP in different eluting systems.

\subsection{Cholesterol binding and adsorption studies}

After the removal of the template molecules, all the cholesterol imprinted polymers were able to select the adsorption of cholesterol from the solution. Thefigure 8shows that the amount of adsorbed cholesterol increased with increasing initial cholesterol concentration on the increase of driving force. The higher initial cholesterol concentration could enhance the adsorption process [26]. The high uptake of cholesterol by HEMA-based MIP may be attributed to the higher degree of hydrophobic interactions prior to polymerization, leading to the creation of more recognition sites in HEMA-based MIP for cholesterol.

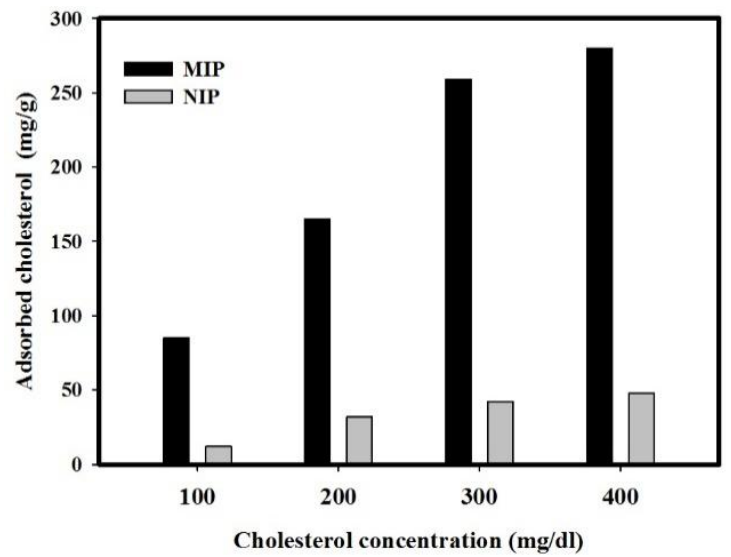

Figure 6:Effect of different cholesterol concentration on cholesterol adsorption $(\mathrm{mg} / \mathrm{g})$ by MIP and NIP after immersion for 24 hours.

\subsection{Selectivity Studies}

The selectivity of MIPs and NIPs toward cholesterol and testosterone.From the figure, it can be observed that, cholesterol MIP has higher binding capacities for cholesterol than for testosterone;MIP bond cholesterol is more selective thantestosterone. Imprinting-induced promotion of binding (IPB) of testosterone to MIP showed that the MIPs had low cross-reactivity with testosterone, Meanwhile, IPB of cholesterol to MIP showed that the MIPs had high crossreactivity with cholesterol and the recoveries of cholesterol on MIP cartridges was higher than that of testosterone.In 


\section{International Journal of Science and Research (IJSR) \\ ISSN (Online): 2319-7064}

Index Copernicus Value (2013): 6.14 | Impact Factor (2014): 5.611

addition, the MIPs has selectivity towardcholesterol than NIPs which has no selectivity for both cholesterol andtestosterone. Cholesterol is a 27-carbon alcohol. It has a hydroxyl group at $\mathrm{C} 3$ position and an eight-carbon branched aliphatic group attached to $\mathrm{C} 17$. Although testosterone has a similar ring structure and shape to cholesterol but it differs from cholesterol by substituting of hydroxyl group at the branched aliphatic group at $\mathrm{C} 17$ position lacking of a methyl group at $\mathrm{C} 19$ position and a carbonyl group at $\mathrm{C} 3$ position [28].Testosterone hasno side chain, and is more hydrophilic than cholesterol.

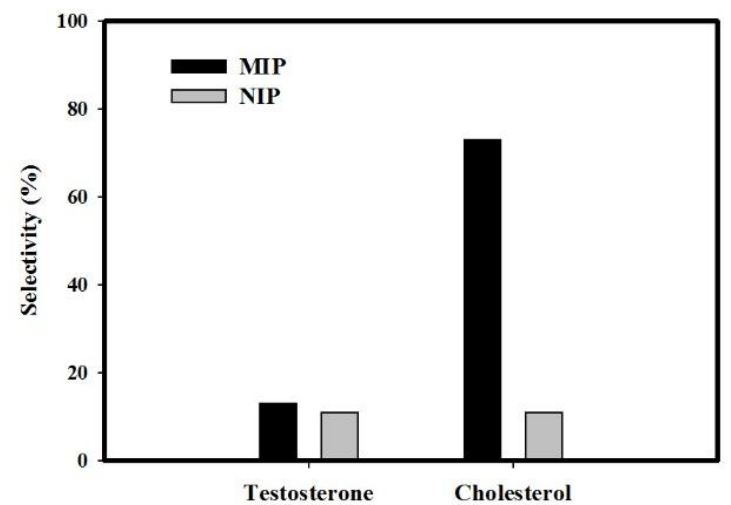

Figure 7: Selectivity of NIPs and MIPs for cholesterol and testosterone

\section{Conclusion}

Selection of the polymerization conditions and template are the most important step to synthesize such Non-covalent imprinted polymers. The attractive target molecule(template) in this work was cholesterol. Carefully synthesized MIP showed ability to absorb cholesterol. Batch adsorption is a simple and effective method to evaluate the imprinting effect.Carefully synthesized MIP showed selective recognition to cholesterol than other steroids.

\section{References}

[1] Sayin, U., Can, C., E Türkkan, Ö Dereli, Ozmen, A., \& H Yüksel 2013. Radiation Damage Centers in Cholesteryl Heptanoate. Acta Physica Polonica A, 124(1): 70-73.

[2] Okazaki, H., Tazoe, F., Okazaki, S., Isoo, N., Tsukamoto, K., Sekiya ,M., Yahagi, N., Iizuka, Y., Ohashi, K., \& Kitamine, T. 2006. Increased cholesterol biosynthesis and hypercholesterolemia in mice overexpressing squalene synthase in the liver. Journal of Lipid Research, 47(9): 1950-1958.

[3] Kugimiya, A., Kuwada, Y., \& Takeuchi, T. 2001. Preparation of sterol-imprinted polymers with the use of 2-(methacryloyloxy) ethyl phosphate. Journal of Chromatography A, 938(1.135-131 :)

[4] Renata Gadzala-Kopciuch, Júlia Ricanyová, \& Boguslaw Buszewski 2009. Isolation and detection of steroids from human urine by molecularly imprinted solid-phase extraction and liquid chromatography. Journal of Chromatography B, 877(11): 1177-1184.

[5] Nóra Adányi \& Mária Váradi 2003. Development of organic phase amperometric biosensor for measuring cholesterol in food samples. Eur Food Res Technol, 218(1): 99-104.

[6] Zhang, R. Z., Li, L., Liu, S. T., Chen, R. M. and Rao, P. F. 1999: Animproved method of cholesterol determination in egg yolk by HPLC. Journal of Food Biochemistry, 23(3): 351-361

[7] Boselli, E., Caboni, M. F., \& Lerker, G. 1997 :Determination of free cholesterol from dried egg yolk by on-line coupling of supercritical fluid extraction with solid-phase extraction.Zeitschrift fur LebensmittelUntersuchung und -Forschung Unters. Forsch. 205(5): 356-359.

[8] Vasapollo, G., Sole, R. D., Mergola, L., Lazzoi, M. R., Scardino, A., Scorrano, S., \& Mele, G. 2011. Molecularly imprinted polymers: present and future prospective. International Journal of Molecular Sciences, 12(9): 5908-5945.

[9] Kofinas, P. \& Kioussis, D. R. 2003. Reactive phosphorus removal from aquaculture and poultry productions systems using polymeric hydrogels. Environmental Science \& Technology, 37(2): 423-427.

[10] Atinafu, D. G. \& Bedemo, B. 2011. Estimation of total free fatty acid and cholesterol content in some commercial edible oils in Ethiopia, Bahir DAR. $J$ Cereals Oilseeds, 2: 71-76.

[11] Joseph H.O.Owino, Omotayo A.Arotiba, Priscilla G.L.Baker, Anthony Guiseppi - Elie, \& Anthony Guiseppi - Elie 2008. Synthesis and characterization of poly (2-hydroxyethyl methacrylate)-polyaniline based hydrogel composites. Reactive \& Functional Polymers, 68: 1239-1244.

[12]Duran, S., D Solpan, \& O Güven 1999. Synthesis and characterization of acrylamideGÇôacrylic acid hydrogels and adsorption of some textile dyes. Nuclear Instruments and Methods in Physics Research Section B: Beam Interactions With Materials and Atoms, 151(1): 196-199.

[13] Kugimiya, A. \& Takeuchi, T. 1999. Effects of 2hydroxyethyl methacrylate on polymer network and interaction in hydrophilic molecularly imprinted polymers. Analytical Sciences, 15: 29-34.

[14]H Kasgöz, S Özgümüs, \& Orbay ,M. 2003. Modified polyacrylamide hydrogels and their application in removal of heavy metal ions. Polymer, 44(6): 17851793.

[15] Singh, L. K., Singh, M., \& Singh, M. 2014. Biopolymeric receptor for peptide recognition by molecular imprinting approach - Synthesis, characterization and application. Materials Science and Engineering: $\boldsymbol{C}, 45$ : 383-394.

[16] Taleb, M. F. A., Abdel-Aal, S. E., El-Kelesh, N. A., \& Hegazy, E. S. 2007. Adsorption and controlled release of chlortetracycline $\mathrm{HCl}$ by using multifunctional polymeric hydrogels. European Polymer Journal, 43(2): 468-477.

[17] Andac, M., Galaev, I. Y., \& Denizli, A. 2013. Molecularly imprinted poly (hydroxyethyl methacrylate) based cryogel for albumin depletion from human serum. Colloids and Surfaces B: Biointerfaces, 109: 259-265.

[18] Yavuz, H., Veyis Karakoç, Deniz Türkmen, Say, R., \& Denizli, A. 2007. Synthesis of cholesterol imprinted 


\section{International Journal of Science and Research (IJSR) \\ ISSN (Online): 2319-7064}

Index Copernicus Value (2013): 6.14 | Impact Factor (2014): 5.611

polymeric particles. International Journal of Biological Macromolecules, 41(1): 8-15.

[19] Shi, Y., Zhang, J. H., Shi, D., Jiang, M., Zhu, Y. X., Mei, S. R., Zhou, Y. K., Dai, K., \& Lu, B. 2006. Selective solid-phase extraction of cholesterol using molecularly imprinted polymers and its application in different biological samples. Journal of Pharmaceutical and Biomedical Analysis, 42(5-549 :) .555

[20] Daksha, A., Jaywant, P., Bhagyashree, C., \& Subodh, P. 2010. Estimation of sterols content in edible oil and ghee samples. Electronic Journal of Environmental, Agricultural and Food Chemistry, 9(10): 1593-1597.

[21] Wang, S., Yu, H., \& Wickliffe, J. K. 2011. Limitation of the MTT and XTT assays for measuring cell viability due to superoxide formation induced by nano-scale $\mathrm{TiO}$ 2. Toxicology in Vitro, 25(8): 2147-2151.

[22] Morrison, W. R. \& Laignelet, B. 1983. An improved colorimetric procedure for determining apparent and total amylose in cereal and other starches. Journal of Cereal Science, 1(1): 9-20.

[23]El Hegazy, S. A. 2008. Gamma radiation sterilization service centre in a developing country: Egypt. Trends in radiation sterilization of health care products.

[24]Basfar, A. A. \& Lotfy, S. 2015. Radiation-crosslinking of shape memory polymers based on poly (vinyl alcohol) in the presence of carbon nanotubes. Radiation Physics and Chemistry, 106: 376-384

[25] Raafat, A. I., Araby, E., \& Lotfy, S. 2012. Enhancement of fibrinolytic enzyme production from Bacillus subtilis via immobilization process onto radiation synthesized starch/dimethylaminoethyl methacrylate hydrogel. Carbohydrate Polymers, 87(2): 1369-1374

[26] Paradkar, M. M. \& Irudayaraj, J. 2002. Determination of cholesterol in dairy products using infrared techniques: 1. FTIR spectroscopy. International Journal of Dairy Technology, 55(3): 127-132.

[27] Gore, M. A., Karmalkar, R. N., \& Kulkarni, M. G. 2004. Enhanced capacities and selectivities for cholesterol in aqueous media by molecular imprinting: role of novel cross-linkers. Journal of Chromatography B, 804(1): 211-221. 